\title{
Networked Gridlock: Students Enacting Complex Dynamic Phenomena with the HubNet Architecture
}

\author{
Uri Wilensky ${ }^{(1)}$ \\ Center for Connected Learning \& Computer-Based Modeling \\ Tufts University \\ Tel: (617) 627-5396 \\ Email: uriw@media.mit.edu \\ Walter M. Stroup \\ Department of Curriculum \& Instruction \\ The University of Texas at Austin \\ Tel: (512) 471-3747 \\ Email:Wstroup@mail.utexas.edu
}

\begin{abstract}
This project brings together two lines of research - the study of complex dynamic systems and the use of participatory simulations as a powerful way into systems modeling-both of which can be enabled and advanced through emerging network technologies. The study of dynamic systems stands as a new form of literacy for all. Participatory Simulations Activities can support new forms of classroom interaction and can serve to catalyze the engagement with dynamic systems modeling as a core feature of the education of all students. To accomplish these goals, we introduce a new architecture, HubNet. HubNet is an open client-server architecture, which enables many users at the "Nodes" (currently TI graphing calculators) to control the behavior of individual objects or agents and to view the aggregated results on a central computer known as the Hub. This network of nodes is integrated with a powerful suite of modeling, analysis and display tools that together give users the capacity to "fly' the system in intuitive mode, to reflect on the emergent result of their simulation and, also, to encode their strategies as rules which the system can then run independently. The HubNet system is being used in several middle and secondary classrooms. An illustrative example of classroom use is presented.
\end{abstract}

Keywords: Simulations, modeling, mathematics education, science education.

\section{Introduction}

In this paper, we describe a new network-based architecture, HubNet, designed for enabling students to engage in participatory simulations of complex dynamic systems. Working together with a commercial partner, we are engaged in an iterative design and test cycle to refine the HubNet system and its associated activities. Early versions of HubNet are in use in classrooms in Boston Massachusetts and Austin Texas. This work is being undertaken under the auspices of the Participatory Simulations Project (PSP) - an NSF-funded collaboration between Tufts University's Center for Connected Learning and The University of Texas at Austin.

In the following sections of this paper, we introduce our project and give an example of its use in school settings. We begin with a brief history of the use of participatory simulations in math/science education, move on to describing the HubNet architecture and an illustrative classroom activity, then outline future directions of the Participatory Simulations Project.

\section{What's a Participatory Simulation?}

Students engaged in participatory simulations act out the roles of individual system elements and then see how the behavior of the system as a whole can emerge from these individual behaviors. The emergent behavior of the system and its relation to individual participant actions and strategies can then become the object of collective discussion and analysis. 
While such participatory role-playing activities have been commonly used in social studies classrooms, they have been infrequently used in science and mathematics classrooms. Our use of the term participatory simulations is intended to refer to such role-playing activities aimed at exploring how complex dynamic systems evolve over time. Our focus is primarily on learning in science and mathematics classrooms. For example, each class member could play the role of a predator or prey in an ecology and engage in a classwide discussion of the resultant global population dynamics. A wide ranging set of sample content areas for participatory simulations include the spread of a disease, the flow of traffic in a grid, the distribution of goods in an inventory system, the diffusion of molecules through a membrane, or the emergence of an algebraic function from a set of points

\section{Why do Participatory Simulations and Emergent Activities Matter?}

A core commitment of this Project is to research the use of participatory simulations as a way into systems dynamics and complexity learning for $A L L$ students. During recent decades, there has been a recognition of the importance of understanding the behavior of dynamic systems-how systems of many interacting elements change and evolve over time and how global phenomena can arise from local interactions of these elements. New research projects on chaos, self-organization, adaptive systems, nonlinear dynamics, and artificial life are all part of this growing interest in systems dynamics. The interest has spread from the scientific community to popular culture, with the publication of general-interest books about research into dynamic systems

It is the stance of the PSP that the study of dynamic systems is not just a new research tool or new area of study for scientists. Our stance is that the study of dynamic systems stands as a new form of literacy for all, a new way of describing, viewing, and symbolizing phenomena in the world. The language of the present mathematics and science curriculum employs static representations. Yet, our world is, of course, constantly changing. This disjunct between the world of dynamic experience and the world of static school representations stands as one source of student alienation from the current curriculum (Chen \& Stroup 1994; Wilensky \& Reisman, 1998).

Research in mathematics/science education and cognitive science (Mandinach \& Cline, 1994) has documented that students have considerable difficulties in making sense of complex systems. In particular, Resnick and Wilensky have documented the considerable difficulties people have in making sense of emergent phenomena, global patterns that arise from distributed interactions, central to the study of complex systems. This constellation of difficulties in understanding emergent phenomena and constructing distributed explanations of such phenomena has been labeled the "deterministic/centralized mindset" (Resnick \& Wilensky, 1993; Wilensky \& Resnick, 1999; Resnick, 1996). Our aim in the PSP and in developing the HubNet system is to be catalytic in helping secondary and post-secondary students move beyond the deterministic/dentralized mindset and advance their understanding of complex dynamic systems. We view the facility with systems thinking, modeling and emergence as a new and necessary form of literacy for our citizenry.

The theoretical and computer-based tools arising out of the study of dynamic systems can describe and display the changing phenomena of science and the everyday world. A core conjecture of the PSP is that the affordances of participatory simulations, as supported by networked modeling and analyses tools discussed below, provide a powerful way into systems related sense-making that can help realize the vision of systems learning for all students.

\section{What's New in the Participatory Simulations Project?}

The list of what is new about the PSP includes the development and use of innovative networked classroom-based technologies to connect learners' evolving intuitions with powerful tools for modeling and analysis; and the pursuit of fundamental research into emergent learning through the use of network-based interactivity. These advances are to be supported by new fundamental research into emergent learning and by developing an innovative, networked technological substrate in close collaboration with a commercial partner, Texas Instruments.

By interconnecting the analyzable artifacts created from learner activity with powerful tools of analysis and modeling the enactive aspects of participatory simulations stand to be deepened and extended. Additionally, learners working in the networked environment make overt and visible their strategies in relation to generating different kinds of emergent behavior. In so doing, these strategies become increasingly well-articulated and refined in ways that scaffold both learner understanding of dynamic systems and the actual use by learners of the tools 
themselves. Through the participation in and analysis of emergent activities, we expect learners to come to see the tools as increasingly useful in helping them to further articulate their insights into the emergent behavior of dynamic systems. These tools enable them to analytically understand these systems, in effect working with the mathematics of change without needing to master the formalisms of differential equations. From the research side of the PSP, the network-based activity will help to make visible learners' ideas and ways of organizing their experiences, which should significantly advance our understanding of these forms of emergent learning.

\section{A Brief History of Participatory Simulations}

The first major instance of which we are aware where a participatory simulation was used in the context of systems dynamics and systems learning was The Beer Game as developed by Jay Forrester and his systems dynamics group at MIT in the early 1960's. There is a significant literature related to The Beer Game and interest in this participatory simulation has been recently revitalized as a result of its appearance in Senge's widely read The Fifth Discipline (1990). The game does much to highlight the ways in which costly unintended behaviors of a system (in this case beer inventory in a distribution system) can emerge from participants attempting to act rationally in their localized role (e.g., as beer retailer, wholesaler, distributor, or producer). A number of other such PSA were developed at this time. One popular PSA, FishBanks (Meadows, 1986) was developed by Meadows as an "interactive, role-playing simulation in which groups are asked to manage a fishing company." Students try to maximize their assets in a world with renewable natural resources and economic competition.

More recently, new classes of so-called "object-based" simulation activities have been developed (Resnick \& Wilensky, 1993; 1998; Wilensky \& Resnick, 1995). In these so-called "StarPeople" activities, participants typically play the role of "ants" in an anthill simulation, moving around the room and exchanging "messages." After participating in these StarPeople activities students observe the emergence of global patterns from their local interactions. These pattern become the objects of reflection and discussion.

\section{Participatory Simulations Activities and Computational Tools}

Throughout much of the fifty-year history of participatory simulations computational technologies have played a central role. The systems dynamics group at MIT developed a class of computational "flight simulators" to be used by individuals and groups of managers to gain experience flying a complex dynamic system like a modern business. More recently, multi-player networked versions of the beer game have been implemented (Coakley et al, 1995 ) and it is now even possible to immerse oneself in a multi-player versions of the game on the internet (Powersim Corporation, 1998). A multi-player calculator-based version of the beer game participatory simulation also has been implemented and used with both school-aged and adult learners (Wilensky \& Stroup, 1998, 1999). Management trainers have argued that there is a need for a tighter coupling between computer simulations and user experience. In possibly the first known use of the term participatory simulations, Diehl (1990) constructed systems that gave users more control over and participation within the simulations by allowing users to input more real word decisions and view output of familiar reports. These simulations were modeled using finite-difference tools like STELLA.

In contrast to the "aggregate" finite-difference computer modeling tools used to analyze simulations like The Beer Game, these simulation activities have been designed to be further explored using object-based parallel computer modeling languages (OBPML) such as StarLogo and StarLogoT (Resnick, 1994; Wilensky, 1995; 1997b). Borovoy, Colella and fellow researchers at MIT (Colella et al, 1998; Borovoy et al , 1996; 1998) have developed wearable computational badges (or "thinking tags") that allow users to move freely while communicating information between badges. Colella (1998) developed, implemented and researched student learning with one of the first instances of a participatory simulation supported by a thin layer of computing technology. Disease propagation models are natural candidates for this kind of participatory simulation and have been implemented by a number of researchers and curriculum developers (Colella et al, 1998; Stor \& Briggs, 1998).

A significant innovation in this project is a commitment to exploring the complementarity of these two fundamental kinds of dynamical systems modeling - aggregate and object-based approaches. This compels a careful attention to a) the relationships between macro- and micro-levels of understanding a system (Chen \& Stroup, 1994; Wilensky, 1993; 1997a); b) thinking in levels (Wilensky and Resnick, 1999); c) systems thinking (Chen \& Stroup, 1994); and d) the analysis of systems like gases (Wilensky, 1994; Wilensky, 1999; Wilensky, Hazzard, and Froemke, 1999). Through the use of participatory simulations and attention to the kinds of constructs learners 
articulate and extend in relation to both the aggregate and object-based modeling environment, we expect to gain deeper insights into how these kinds of distinct but inter-related forms of analyses interact and complete one another.

\section{What is HubNet?}

HubNet is the name we have given to a new architecture we have designed to give students the experience of participating as elements in a simulation of a complex dynamic system. HubNet is an open client-server architecture, which enables many users at the "Nodes" to control the behavior of individual objects or agents and to view the aggregated results on a central computer known as the "Hub". This network of nodes is integrated with a powerful suite of modeling, analysis and display tools that, together, give the capacity to "fly" the system in intuitive mode, to reflect on the emergent result of the simulation and to encode student strategies as rules which the system can then run independently.
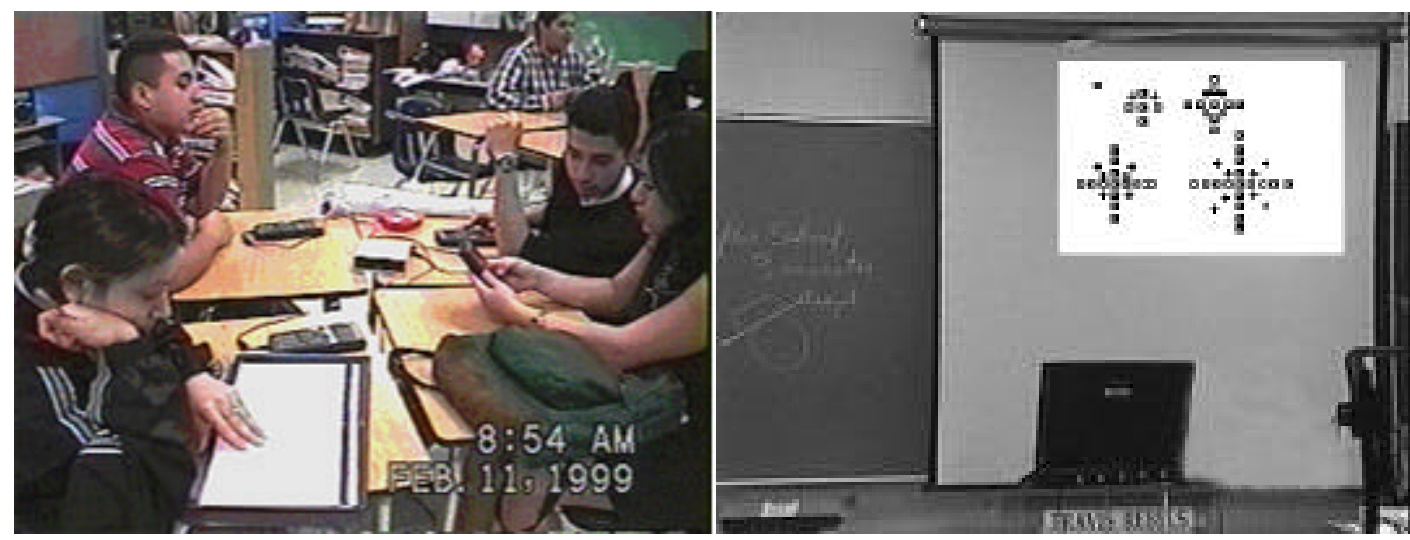

Figure 1. Students engaged in participatory simulation supported by HubNet calculator network. On the left each student sees her/his own calculator view. On the right is the projected classroom display of the emergent result.

The HubNet architecture is being developed in stages. At present, a workable minimal subset of functionality is implemented. This subset consists of 1) a suite of networked graphing calculators called Classnet (or HubCalc) developed in concert with our commercial partner, Texas Instruments. 2) a server, which talks to the Classnet network and 3) an object-based parallel modeling language, N-Logo, which is an enhanced port of the StarLogoT language that enables users to build object-based models of systems consisting of thousands of distributed elements. We call this three-component HubNet sub-system ClassLogo. Future versions of HubNet will integrate aggregate modeling languages, such as STELLA, facilitating a dialogue between object-based and aggregate approaches. Many more analysis and display tools will also be as well as hooks allowing a much wider array of node hardware including arbitrary Internet hosts.

A potential barrier to wide-spread adoption of networked activities is the difficulties in authoring new PSA. Our Java-based development effort of N-Logo extends the object-based modeling capabilities of StarLogoT by having the N-Logo language also serve as a scripting language for the creation of HubNet-based participatory simulations. Just as object-based models are extensible (Wilensky, 1997a, 1999), the network-based emergent activities created in N-Logo will be extensible. Under the HubNet design, the parallelism of StarLogoT and N-Logo as modeling environments is being significantly extended to also serve as a way of coordinating and authoring activities for a space of networked computing devices (nodes).

HubNet is designed with the assumption that the nodes have significant resident functionality (at least that of a programmable graphing calculator). The network layer implements flexible communication protocols that include the ability to upload and download data sets, upload and download program (e.g., applets), monitor keypresses at the hand-held level, support real-time interaction as in network computer games, and form collaborative groups of various sizes (e.g., peer to peer, small groups, and whole class modes). The HubNet hardware includes an up-front computer (the "hub") capable of addressing the network of nodes and a display capability (e.g., a projection system) that enables an entire class to view the simulation. 
As described earlier, the current working subset of HubNet, ClassLogo, uses the prototype TI Classnet system (2) consisting of a suite of networked graphing calculators. The resident functionality of the calculator, including capabilities of interacting with real world devices such as sensors and motors, CBLs and CBRs, allows for a wide range of PSA to be implemented in the classroom.

ClassNet supports fully networked modes of interaction with and among learners. While synchronization between the data on the hand-helds and the Hub is supported in this model, the model can also support on-going, real-time interactivity and exchange. This fully-networked HubNet system is intended to support a range of different topologies for collaboration among learners including point-to-point, small group and whole class interaction. This more inclusive range of interactivity and richly textured forms of collaboration cannot be supported in models based exclusively on the synchronization model of uploading data from after a simulation has been run.

\section{HubNet in the Classroom - An Illustrative Example: Gridlock}

Participatory simulations stand to introduce fundamentally new and significant forms of reasoning and insight to school-based curricula. Elsewhere we have presented examples of where participatory simulations allow students to learn concepts at the core of the traditional curriculum in exciting new ways (See Function Activity in Wilensky \& Stroup, 1999). The following example illustrates the use of participatory simulations to introduce fundamentally new ideas into classroom learning. We start with a scenario that is meaningful and where the class as whole has a goal they are aiming to accomplish. By playing a role in the simulation, the knowledge students develop is even more situated and embodied than it would be from just being presented with the scenario alone. Students are not simply "active" in a participatory simulation, they are enactive and this stands to improve both student motivation and understanding. The sense of ownership is further extended by the fact the students analyze the results they helped to create. A network of handheld devices supports real-time interaction in this simulation. Real-time interaction represents a significant improvement in efficiency and in the range of participatory simulations that can be supported over simply uploading data after a simulation has been completed (Wilensky \& Stroup, 1998; Colella, 1998).

The scenario the students are presented with is this: The mayor of the City of Gridlock has commissioned the class to improve the traffic situation in the city. The traffic simulation is projected in front of the class and begins running with cars heading through the city. The city starts off with no traffic lights. Graphs depicting the average speed of the cars passing through the city and the cars' wait time are also plotted (not shown). On first impressions, it looks like everything is running smoothly for this city (Figure 2). The average velocity is relatively high and the average wait time is close to zero.

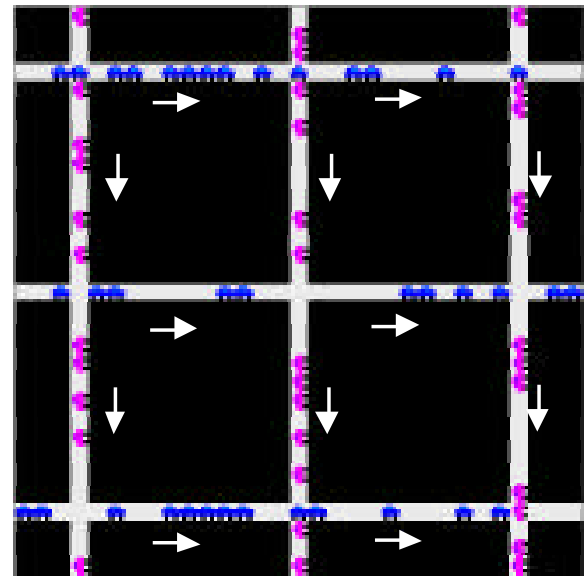

Figure 2. Traffic flow with no lights.

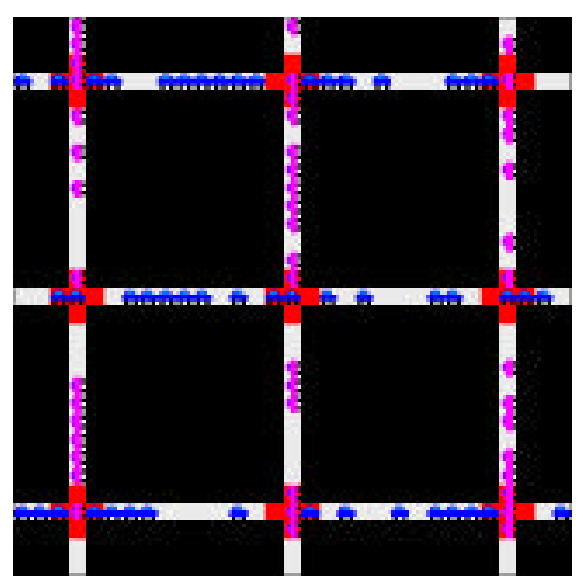

Figure 3. Accidents quickly result.

The problem is that the initial simulation doesn't keep track of when cars are occupying the same location on the grid. In real life, two (or more) cars trying to occupy the same location is called a crash. If the upfront simulation now shows these crashes - denoted with red crosses - every intersection quickly has a red cross on it (Figure 3 ). 
Traffic comes to a complete standstill. The initial introduction of the participatory simulation is completed by adding a single traffic light. When the simulation is run again, the teacher can turn the lights at the intersection green and red using a switch on the N-Logo interface (green in one direction means red in the other). In addition to illustrating the use of the switch, it also soon become apparent that accidents re-emerge at every intersection except the one. the one with the traffic light.

Now traffic lights are placed at every intersection and students can control these lights using the up-arrow keys on their calculators. After each student locates his/her intersection and practices changing the lights, cars are introduced. The good news is that there are no accidents in the City of Gridlock. The bad news is that traffic is backing up all over the city (Figure 4), the average speed through the city is low, the average wait time is high, and the Mayor would not be pleased. Students then explore various ways of coordinating their lights. Very subtle questions about logic, timing, phase and synchronization are engaged as students struggle both to create ways of talking about the traffic that are meaningful and to implement strategies making use of this language.

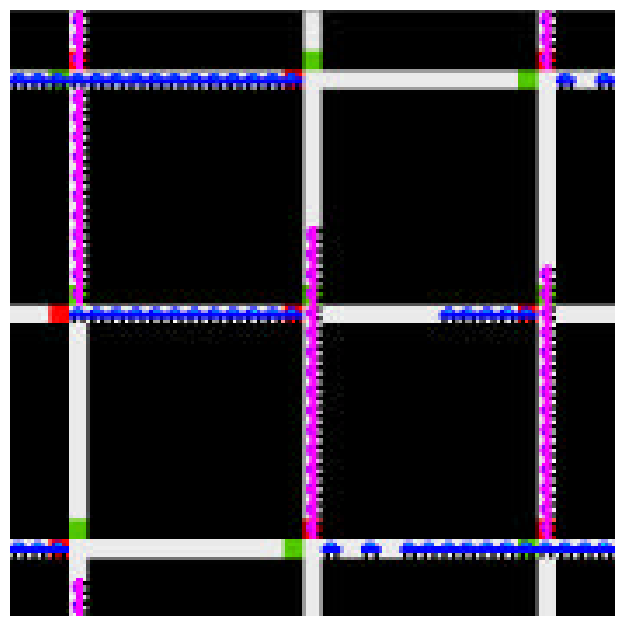

Figure 4. Uncoordinated Traffic

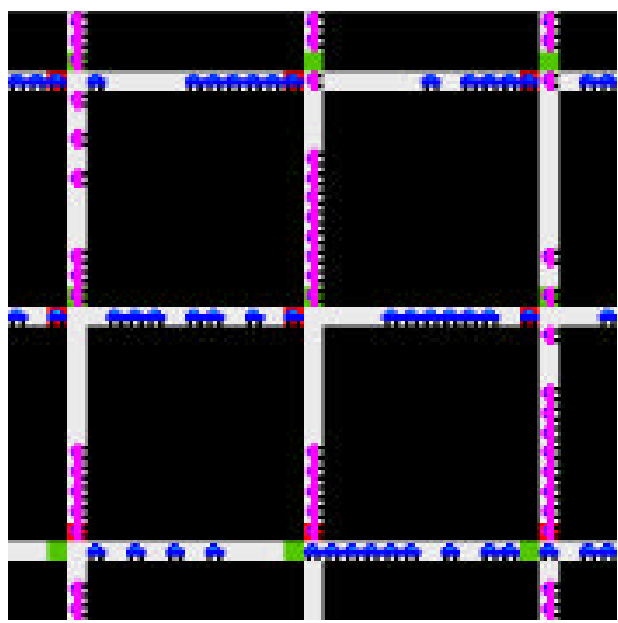

Figure 5. Lights synchronized

One of the strategies is to synchronize the lights with a phase shift. In Figure 5. the lights in the top row turned green in the same direction together, then the lights in the second row waited a few seconds (phase shift) and turned green. This pattern cascades downward as traffic flow in that direction is synchronized. Using the network, data from the various trials can be handed back out to the students. This coordinated approach does improve traffic flow.

It is worth emphasizing that students will actually develop a range of strategies in this participatory simulation. For example, students often develop a "traffic cop" strategy. Each "light" (traffic cop) would look to see in which direction there were more cars and let that direction go. This strategy has significant counter-intuitive consequences. Other students have begun to explore the idea of "smart cars" where accidents might be avoided if the cars had enough built-in intelligence to figure out if a car coming from the side was going to hit it. The smart car would then take steps to avoid the accident and might be able to do that without having to come to a complete stop (as with a traffic light). As part of preparing a report of recommendations to the mayor of Gridlock, the student strategies need to be analyzed. Using the network, data can be easily collected and exchanged. The HubNet architecture readily supports the distributed analyses of these different student strategies.

For the purposes of analysis and comparison, the handheld devices (calculators) have significant resident functionality. This means mathematically meaningful tools are available to every student (graphs, tables, histograms, etc.). The flow of information and the location of the tools of analysis do not remain "centralized" in this model, but instead a multi-directional (student to student, teacher to student, student to teacher, etc.) flow and exchange of analyses is supported. In the traffic example, various metrics for measuring the improvement in traffic flow can be developed by students and a set of final recommendations can be developed as a report (or collection of reports) to the mayor. The reports will incorporate elements of both object-based and aggregate analyses. For students, the question of "what is math for" would be answered in a way that is interesting and engaging precisely 
because participatory simulations are complex. In this way they are more like the highly complex, dynamic world students experience outside of school and outside of traditional math and science education.

\section{Future Directions}

As indicated above, the HubNet architecture is in a preliminary stage. Significant project resources are allocated to developing HubNet and completing the fully networked architecture. Alongside this iterative design research, we will continue to conduct both implementation and curricular research with successive versions of HubNet. We have begun to design and test a set of PSA using ClassLogo that make use of sophisticated new content domains. This fundamental research is being carried out in economically challenged inner-city schools. Significant resources from the PSP Project are going toward site-based support and innovation. We are working alongside the teachers in targeting and implementing network-based participatory simulations that can transform students' understanding of core concepts of the current curriculum (e.g. the concept of function) even as fundamentally new systems-understandings and content areas are introduced.

In this context, we seek to gain a better understanding of how a PSA can significantly advance student understanding of the unfolding dynamics of systems. We hope to shed light on how learners' intuitive understandings and ways of responding interact with rule-based, embodied (e.g., StarLogoT, N-Logo) and aggregate (e.g., STELLA) modeling environments. Through this design, implementation and curricular research, we hope to further the goal of advancing systems related understanding for all students.

\section{Acknowledgements}

The preparation of this paper was supported by the National Science Foundation (Grants REC-9814682, REC9632612), The ideas expressed here do not necessarily reflect the positions of the supporting agency.

\section{Endnotes}

(1) This work is a collaboration. There is no primary author.

\section{References}

Borovoy, R., McDonald, M., Martin, F., \& Resnick, M. (1996). Things that Blink: Computationally Augmented Name Tags. IBM Systems Journal, 35(3).

Chen, D., \& Stroup, W. (1993). General Systems Theory: Toward a conceptual framework for science and technology education for all. Journal for Science Education and Technology

Colella, V., Borovoy, R., and Resnick, M. (1998) Participatory Simulations: Using Computational Objects to Learn about Dynamic Systems. Proceedings of the Computer Human Interface (CHI) '98 conference, Los Angeles, April 1998.

Forrester, J. W. (1968). Principles of Systems. Norwalk, CT: Productivity Press.

Jackson, S., Stratford, S., Krajcik, J., \& Soloway, E. (1996). A learner-centered tool for students building models. Communications of the ACM, 39(4), 48-49.

Mandinach, E. B., \& Cline, H. F. (1994). Classroom dynamics: Implementing a technology-based learning environment. Hillsdale, NJ: Lawrence Erlbaum Associates

Meadows, D. (1986). Fish Banks, LTD. In Durham, NH: Institute for Policy and Social Science Research. http://www.unh.edu/ipssr/index.html/ipssr/lab/fishbank.html

Papert, S. (1980). Mindstorms: Children, computers, and powerful ideas. New York: Basic Books.

Resnick, M. (1994). Turtles, Termites and Traffic jams. Explorations in massively parallel microworlds. Cambridge, MA: MIT Press.

Resnick, M., \& Wilensky, U. (1998). Diving into complexity: Developing probabilistic decentralized thinking through role-playing activities. Journal of the Learning Sciences, 7(2), 153-171.

Resnick, M., \& Wilensky, U. (1993). Beyond the deterministic, centralized mindsets: New thinking for new sciences. Presented at the annual conference of the American Educational Research Association, Atlanta, GA.

Richmond, B., \& Peterson, S. (1990). Stella II. Hanover, NH: High Performance Systems, Inc.

Roberts, N. (1978). Teaching dynamic feedback systems thinking: an elementary view. Management Science, 24(8), 836-843. 
Senge, P. (1990). The Fifth Discipline. New York: Doubleday/Currency.

Stor, M. \& Briggs, W. (1998). Dice and disease in the classroom. The Mathematics Teacher, v. 91, no. 6. Pp 464468.

Stroup, W. (1995). Dynamics and calculus for the young learner. Teacher's Lab, 16-18.

Stroup, W. (1996). Embodying nominalist constructivism: Making graphical sense of learning the calculus of how much and how fast. Doctoral Dissertation. Harvard Graduate School of Education. Cambridge, MA.

Wilensky, U. \& Stroup, W. (1999). Learning through Participatory Simulations: Network-based Design for Systems Learning in Classrooms. Proceedings of the Computer Supported Collaborative Learning Conference, Stanford University, December 1999.

Wilensky, U \& Stroup, W. (1998). The Root Beer Game.

Wilensky, U \& Stroup, W. (1999). The Distribution Simulation.

Wilensky, U. (1999). GasLab-an extensible modeling toolkit for exploring micro- and macro- views of gases. In N. Roberts, W. Feurzeig, \& B. Hunter (Eds.), Computer modeling and simulation in science education. Berlin: Springer Verlag.

Wilensky, U. \& Resnick, M. (1999). Thinking in Levels: A Dynamic Systems Perspective to Making Sense of the World. Journal of Science Education and Technology. Vol. 8 No. 1.

Wilensky, U. \& Resnick, M. (1995). New Thinking for New Sciences: Constructionist Approaches for Exploring Complexity". Presented at the annual conference of the American Educational Research Association, San Francisco. Ca.

Wilensky, U. \& Reisman, K. (1998). Learning Biology through Constructing and Testing Computational Theories - an Embodied Modeling Approach. In Y. Bar-Yam (Ed.), Proceedings of the Second International Conference on Complex Systems. Nashua, NH: New England Complex Systems Institute.

Wilensky, U. (1997a). What is Normal Anyway? Therapy for Epistemological Anxiety. Educational Studies in Mathematics. Volume 33, No. 2. pp. 171-202

Wilensky, U. (1997b). StarLogoT. http://www.ccl.tufts.edu/cm/starlogot/ 